\title{
Robustness to the Loss of Multiple Nodes in the Localizability of Sensor Networks *
}

\author{
S. Alireza Motevallian, Changbin Yu, Brian D.O. Anderson ** \\ * Research School of Information Sciences and Engineering, The \\ Australian National University \\ ** Research School of Information Sciences and Engineering, The \\ Australian National University and National ICT of Australia \\ e-mail: \{alireza.motevallian,brad.yu,brian.anderson\}@anu.edu.au
}

\begin{abstract}
In the studies on the localization of wireless sensor networks (WSN), it has been shown that a network is in principle uniquely localizable if its underlying graph is globally rigid and there are at least $d+1$ non-collinear anchors (in $d$-space). The high possibility of the loss of nodes or links in a typical WSN, specially mobile WSNs where the localization often needs to be repeated, enforces to not only have localizable network structures but also structures which remain localizable after the loss of multiple nodes/links. The problem of characterizing robustness against the loss of multiple nodes, which is more challenging than the problem of multiple link loss, is being studied here for the first time, though there have been some results on single node loss. We provide some sufficient properties for a network to be robustly localizable. This enables us to answer the problem of how to make a given network robustly localizable. We also derive a lower bound on the number of the links such a network should have. Elaborating it to the case of robustness against the loss of up to 2 nodes, we propose the optimal network structure, in terms of the required number of distance measurements.
\end{abstract}

Keywords: Robust Localizability, Localization, Wireless Sensor Networks, Global Rigidity.

\section{INTRODUCTION}

Knowing the location of the nodes in a wireless sensor network is critical, as in many applications, the interpretation of the data and decision making is impossible without knowing the position of the detected event. The mobility or unplanned deployment of the nodes in a WSN necessitates a localization technique which can be frequently executed. A variety of techniques are proposed in the literature. Among them, there are schemes in which only a small number of special nodes (called anchors) have their positions known a priori (Aspnes et al., 2006), sometimes because they are GPS-equipped. Then by obtaining a set of distance measurements between enough pairs of ordinary nodes, an algorithm determines the node positions, using the distances and anchor location data; the process is called network localization.

There is a fundamental question in network localization that need to be answered prior to the localization process: what structure should a network have, in order to be localizable? It is important to see beforehand if the network is localizable, as it is a waste of effort to seek to localize a network which is actually not localizable. This question is answered in (Aspnes et al., 2006) with the help of recent results from graph theory including the concept

\footnotetext{
* This work is supported by USAF-AOARD-10-4102. S. A. Motevallian is supported by National ICT Australia (NICTA). C. Yu is supported by the Australian Research Council (ARC) through an Australian Postdoctoral Fellowship under DP- 0877562. B.D.O. Anderson is supported by the ARC and NICTA.
}

of Global Rigidity. It is proved there that a network is uniquely localizable if and only if its underlying graph is globally rigid and there are at least $d+1$ non-collinear anchors in $d$-space $(d \in\{2,3\})$. The underlying graph $G(V, E)$ of a network is the one in which there is a vertex corresponding to each network node in the vertex set $V$ and two vertices are connected via an edge in the edge set $E$ if the distance between the corresponding nodes is known (Note that in modeling a network, the graph itself does not contain the length data). A realization of a graph with associated length data is an assignment of the vertices of the graphs to points in $\mathbb{R}^{d}$ such that the distance between points corresponding to adjacent vertices in the graph equals the distance associated with the corresponding edge of the graph. A graph is called globally rigid if all of its realizations in the $d$-space are congruent, i.e. can be obtained from another realization of the graph only by a combination of reflections, rotations and translations of the whole graph. It is a nontrivial result of the theory that global rigidity is a property determinable from the graph alone (i.e. without the distance set) provided that the distances correspond to generic node positions (e.g. collinearities are likely to be excluded).

One of the most challenging issues in sensor networks is the high possibility of failures either in communication links or sensor nodes themselves, due to different causes, e.g. signal jamming, obstacle, power depletion, mechanical failure, etc. This may result in changes to the structure of the network (and also in the underlying graph). In other words, such failures may cause a previously uniquely 
localizable network to become non-localizable; in a mobile network where re-localization must occur due to the motion, this is especially serious. It is obvious that coping with a single node loss is more demanding than a single link loss as removal of any node also results in the removal of all of its incident links. The solution to the problem of securing tolerance against node loss is to introduce some sort of redundancy in the distance measurements (links), i.e. having network structures which are robust against the loss of some nodes and/or communication links. Despite the importance of this robustness property, only those structures which are robust against the loss of a single node appear to have been studied up to now(Summers et al., 2008). In ( $\mathrm{Yu}$ and Anderson, 2008) some general properties of rigid (a simpler property than global rigidity) which remain rigid after the loss of nodes/links are studied. This work is extended further in (Yu et al., 2010) for sensor network localization, concerning both node loss and link loss tolerance. The authors generalized the notion of redundant rigidity to $(p, q)$-rigidity: the ability to retain (global) rigidity given the loss of any $p-1$ nodes (including their incident links) and also any further $q-1$ links. However, they only established properties associated with redundancy (robustness) under the loss of any $q-1$ links $((1, q)$-rigidity). As is argued in (Yu et al., 2010), characterizing $(p, 1)$-rigidity (redundancy under loss of any $p-1$ vertices) and $(p, q)$-rigidity are still open problems.

In this paper, the problem of robust localizability against the loss of multiple nodes in $2 \mathrm{D}$ is being studied for the first time, through proposing structures for underlying graph which remain globally rigid after the loss of $p$ vertices. We also argue briefly in Section 3.1 that for networks defined by random geometric graphs (a common assumption for large scale sensor networks), formulae are available indicating a minimal transmission radius ensuring the robust localizability property. To elaborate on how the obtained results can be used, we study the case where $p=2$ and suggest a class of graphs which are robust under the loss of up to 2 vertices. Such graphs are, as we show in the paper, optimal in terms of the number of distance measurements they requires. This is a starting point for further studies of such structures for general $p$.

In this work, we always assume the ambient space to be $2 \mathrm{D}$, unless explicitly noticed. We also assume that there are $p+3$ anchors in the network so that after removing any $p$ nodes there are still 3 anchors in the network. The structure of the paper is as follows. In Section 2, the required background is reviewed. Section 3 contains the main contribution of the paper. Finally, concluding remarks are made in Section 4.

\section{BACKGROUND}

In this section we recall some definitions and properties of (minimal) rigidity, redundant rigidity and global rigidity and followed by the general notion of redundant rigidity. For a formal definition and detailed introduction to rigidity and global rigidity please refer to (Graver et al., 1993; Connelly, 2005).

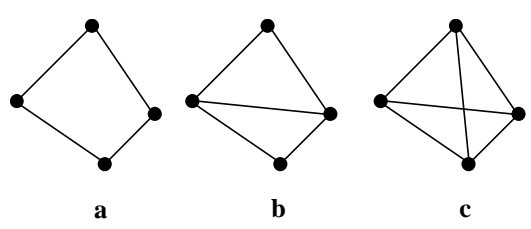

Figure 1. (originally from (Yu and Anderson, 2009)) Realization of a graph in $2 \mathrm{D}$ that is (a) non-rigid, (b) minimally rigid and (c) redundantly rigid (also globally rigid).

\subsection{Rigidity, Minimal Rigidity and Redundant Rigidity}

Assume in a realization of an underlying graph in an ambient space ( $2 \mathrm{D}$ or $3 \mathrm{D})$, each point is a revolute joint and each edge is a solid bar with an specified length. This framework (a term commonly used in rigidity theory), and therefore the underlying graph, is called rigid, if under any motion of the framework in the space, the distance between each pair of points remains constant over time, no matter whether there is or is not an explicit edge connecting them (see (Graver et al., 1993) for a more precise definition). The extension of the term rigid to refer to the graph is valid since it can be shown that if a realization of a graph is rigid for one set of length, a realization for almost any length set will also be rigid. A graph is minimally rigid if it is rigid and removing any one of the edges results in a nonrigid graph (Figure 1b). In (Laman, 1970), it is proved that every rigid graph contains a minimally rigid subgraph with the same vertex set. A graph is termed redundantly rigid, if it is rigid and after removal of any edge, it still remains rigid (Figure 1c).

\subsection{Global Rigidity}

A graph is termed globally rigid, if any two of its realizations in the space are congruent, i.e. each realization can be obtained from any other realization only by a combination of reflections, translations and/or rotations of the whole graph (Figure 1c). The following theorem from (Jackson and Jordan, 2005) gives a characterization of globally rigid graphs in $2 \mathrm{D}$.

Theorem 1 . The graph $G=(V, E)$ with $|V| \geq 4$ is globally rigid, if and only if it is 3 -connected and redundantly rigid.

A graph is said to be $k$-connected if it is connected and after removal of any set of up to $k-1$ vertices, it still remains connected, see (Nagamochi and Ibaraki, 2008).

\subsection{Generalizing Redundant (Global) Rigidity}

The notion of redundant rigidity can be generalized to the loss of $k$ edges and/or $k$ vertices (Yu and Anderson, 2009). A graph is termed $k$-edge rigid if after deletion of any set of up to $k-1$ edges, a rigid graph always results. With the same notion a graph is $k$-vertex rigid if after deletion of any set of up to $k-1$ vertices, the resulting graph is still rigid. Similarly, a graph is called $k$-edge ( $k$-vertex) globally rigid if after deletion of any set of up to $k-1$ edges (vertices), a globally rigid graph always results ( $\mathrm{Yu}$ et al., 2010). The focus of this work is on $k$-vertex global 
rigidity. In this paper, to avoid near trivialities and because the case is actually special in terms of the probable results, we assume that $G=(V, E)$ is not a complete graph (there is an edge between every vertex pair) as it is obvious that the complete graph $K_{l}$ is $(l-2)$-vertex globally rigid and measuring the distances between all pairs of the vertices in the graph (which resulted in a complete underlying graph) is inefficient in practice.

Let us review a few properties of $k$-vertex globally rigid graphs. Lemma 2 is similar to Corollary 2 in ( $\mathrm{Yu}$ and Anderson, 2009), but considers $k$-vertex global rigidity instead of $k$-vertex rigidity.

Lemma 2. If $G=(V, E)$ is $k$-vertex globally rigid, then it is $(k+2)$-connected. Also, each vertex $v \in V$ has degree at least $k+2\left(\delta_{G}(v) \geq k+2\right.$, where $\delta_{G}(v)$ is the degree of vertex $v$ ) and the number of vertices satisfies the inequality $|V| \geq k+3$.

Proof. To prove $(k+2)$-connectivity by obtaining a contradiction, suppose $G$ is not $(k+2)$-connected. Then there exists a cut set, say $S$, where $|S| \leq k+1$. It is obvious (from the definition) that if $|V|<k-1$ then $G$ cannot be $k$-vertex globally rigid. Now let $U \subset S$ be any arbitrary set of vertices with the condition that $|U|=k-1$. According to the definition of $k$-vertex global rigidity, $G \backslash U$ is still globally rigid. However, $T=S \backslash U$ is a cut-set in $G \backslash U$ and has $T=|S \backslash U| \leq 2$ vertices. This means that $G \backslash U$ is not 3 -connected and therefore $G \backslash U$ is not globally rigid. This contradiction implies that $G$ is $(k+2)$-connected.

The $(k+2)$-connectivity implies $\delta_{G}(v) \geq k+2$ for any $v \in V$. Otherwise, there is a set $U$ of up to $k+1$ vertices (the neighbors of a vertex $v$ with $\delta_{G}(v) \leq k+1$ ) whose removal makes the graph disconnected. It follows that $|V| \geq k+3$ holds as there should be at least $k+3$ vertices in $V$ so that every vertex has a degree of at least $k+2$.

A graph is called minimally $k$-vertex globally rigid, if it is $k$-vertex globally rigid but after removing any one of the edges the resulting graph is no longer $k$-vertex globally rigid. It is not hard to argue that (a) $k$-vertex globally rigid graphs exists for any positive $k$ (we present a particular construction in section 3.2 below) and (b) given any $k$ vertex globally rigid graph, a subgraph can be obtained by edge removal which is minimally $k$-vertex globally rigid. Thus the concept is not an empty one.

In (Jordan and Szabadka, 2009) it is shown that there is an operation, called 1-extension (or edge-splitting), which can grow any globally rigid graph by 1 vertex in $2 \mathrm{D}$. In this operation, an edge $(u, v) \in E$ is removed from $E$ and a new vertex $z$, is connected to both $u$ and $v$ and an arbitrary third vertex $w \in V, w \notin\{u, v\}$.

Based on Theorem 1.2 in (Jordan and Szabadka, 2009), the number of edges in a minimally globally rigid graph (the graph $G=(V, E)$ which is globally rigid and $\forall e \in E$ $G^{\prime}=(V, E-e)$ is not globally rigid) is:

Lemma 3. If $G=(V, E)$ is a minimally globally rigid graph on $|V| \geq 4$ vertices, then $|E|=2|V|-2$ always holds.

In the case of standard global rigidity, the definition of minimal global rigidity is proved (Theorem 1.2 (Jordan

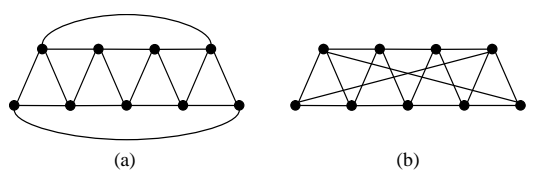

Figure 2. (originally from (Servatius, 1989)) Examples of the 2 possible partition of edge set for strongly minimal 2-vertex rigid graphs: (a) the degree 3 vertices are adjacent, (b) degree 3 vertices are non-adjacent

and Szabadka, 2009)) to be equivalent to an alternative statement: a rigid graph is called minimally globally rigid if it has the minimum number of possible edges $(|E|=2|V|-$ 2 ) among all globally rigid graphs with the same number of vertices. For $k$-vertex global rigidity, these two notions are no longer equivalent; there are some graphs which are minimally 2-vertex globally rigid but the number of edges is not the minimum possible one among such graphs with the same vertex count. This property leads us to two different notions: strongly minimal and weakly minimal $k$-vertex global rigidity (this notion is adapted from the notion of strongly/weakly minimal 2-vertex rigidity in (Summers et al., 2008)).

- A $k$-vertex globally rigid graph is said to be strongly minimal if it has the minimum possible number of edges (over all $k$-vertex minimally globally rigid graphs) on a given number of vertices.

- A $k$-vertex globally rigid graph is said to be weakly minimal if it has more than the minimum possible number of edges on a given number of vertices, but has the property that removing any edge destroys $k$ vertex global rigidity.

The following results of (Servatius, 1989) characterized the structure of strongly minimal 2-vertex rigid graphs for the first time. Due to their relevance and later use, we restate them here. Figure 2, illustrates examples of strongly minimal 2-vertex rigid graphs with each of the two possible types.

Lemma 4. (Lemma 1 of (Summers et al., 2008)) If $G=$ $(V, E)$ is a 2-vertex rigid graph on 5 or more vertices, then $|E| \geq 2|V|-1$.

Theorem 5. (Proposition 1 of (Servatius, 1989)) Let $G=$ $(V, E)$ be a strongly minimal 2-vertex rigid graph on 5 or more vertices. Then $G$ has exactly 3 vertices with degree 3 and the remaining vertices have degree 4 , which implies $|E|=2|V|-1$.

Theorem 6. (Theorem 3.1 of (Servatius, 1989)) A graph $G=(V, E)$ with $|V| \geq 5$ is strongly minimal 2-vertex rigid if and only if $G$ has exactly two vertices of degree 3 and there is a partition of the edge set $E$

$$
E=E_{1} \cup E_{2} \cup \ldots \cup E_{k}
$$

such that the graph induced by $E \backslash E_{i}$ is minimally redundantly rigid (i.e. the removal of any edge destroys redundant rigidity) for all $i$, and either

- $E_{1}$ and $E_{2}$ are the edges incident to the two nonadjacent vertices of degree 3 , respectively, and $E_{i}$ is a single edge for $3 \leq i \leq k$, or

- $E_{1}$ is the union of the edges incident to the two adjacent vertices of degree 3 , and $E_{i}$ is a single edge for $2 \leq i \leq k$. 


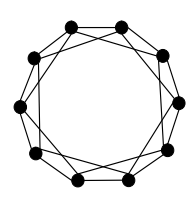

Figure 3. A Strongly Minimal 2-Vertex Globally Rigid graph of size $10\left(C_{10}^{2}\right)$.

The following theorem from (Summers et al., 2008) characterizes strongly minimal 2-vertex globally rigid graphs (Figure 3).

Theorem 7. (Theorem 10 in (Summers et al., 2008)) The graph $G=(V, E)$ of 5 or more vertices is strongly minimal 2-vertex globally rigid if and only if the following conditions hold:

- $|E|=2|V|$

- $G$ is 4-connected

- $G$ is redundantly strongly minimal 2-vertex rigid

A redundantly strongly minimal 2-vertex rigid graph is one in which after removal of any edge, the result is strongly minimal 2-vertex rigid. Such a graph is obtained and can only be attained by joining the vertices of degree 3 in a strongly minimal 2-vertex rigid graph whose vertices of degree 3 are not adjacent. Figure 2 depicts examples of 2 possible configurations for strongly minimal 2-vertex rigid graphs. If in Figure 2(b), we connect the vertices of degree 3 (which are not adjacent), the result is a redundantly strongly minimal 2-vertex rigid graph.

\section{RESULTS}

In this section we start by studying some sufficient conditions for $k$-vertex global rigidity (Section 3.1 ). This will enable us to propose localizable structures which are robust against the loss of up to $k-1$ vertices. Then, from a different perspective, the structure of $k$-vertex globally rigid graphs is studied and some necessary conditions are obtained (Section 3.2). To elaborate this result, the case of 3-vertex global rigidity is considered in more detail and a general class of structures which are strongly minimal 3vertex globally rigid are introduced (Section 3.3 ). We conclude this section by comparing these results on strongly minimal 3-vertex global rigidity and the result that can be obtained by the sufficient condition in Section 3.1.

\subsection{Sufficient Condition for k-Vertex Global Rigidity}

The notion of $k$-connectivity has been well studied in the literature and there are efficient algorithms to check this property on a given graph (Nagamochi and Ibaraki, 2008). The idea here is to identify a $(k+j)$-connectivity condition which is sufficient to ensures $k$-vertex global rigidity. It is proved in (Lovasz and Yemini, 1982) that in 2D, 6connectivity implies rigidity and 6 is the least possible number for this condition ( $k$-connectivity with $k<6$ is not sufficient for rigidity). Recent extension to this work (Jackson and Jordán, 2009) showed the sufficiency of this condition for global rigidity as well.

Theorem 8. (restatement of Theorem 1.2 in (Jackson and Jordán, 2009)) Suppose $G=(V, E)$ is a 6-connected graph in 2D. Then it is 2-edge globally rigid (removing any edge results in a globally rigid graph).

We can extend this result to the case of $k$-vertex global rigidity, as the following theorem shows:

Theorem 9. Assume that $G=(V, E)$ is a $(k+5)$-connected graph. Then $G$ is $k$-vertex globally rigid.

Proof. Let $U \subset V$ be any set of vertices with $|U|=k-1$. Since $G$ is $(k+5)$-connected, it is obvious that $G^{\prime}=G \backslash U$ is 6 -connected $(k+5-(k-1)=6)$. Therefore, $G^{\prime}$ is globally rigid. Since $U$ was chosen arbitrarily, we conclude that $G$ is $k$-vertex globally rigid.

This result is very important as it reduces the more complicated and unfamiliar property of $k$-vertex global rigidity to a well-known and easier to study property of $(k+5)$-connectivity. Specifically for large-scale random wireless networks (where the nodes are assumed to be Poisson distributed with a certain density, and they have common transmission range), it is shown in (Wan and Yi, 2004) that asymptotically, i.e. as the node count tends to infinity, there is a relation between the transmission range and a $k$-connectivity property (for any $k$ ) of a wireless sensor network, i.e. by increasing the transmission power of the nodes in a random network above an specific threshold, the network becomes $k$-connected (The threshold is NOT of the same order as the diameter of the network, a situation which trivially would ensure $k$-connectivity). This idea tied to Theorem 9 answers the question of "given a wireless sensor network, how we can make it robustly localizable":

Theorem 10. Assume a network of wireless sensor nodes. As the node count tends to infinity, there exists a critical transmission radius (NOT of the order of the network diameter), say $r$, such that by increasing the transmission range of every node above it, the network becomes $k$-vertex globally rigid (robustly localizable against the loss of up to $k-1$ vertices).

Finding the value of $r$ for various $k$ is beyond the scope of this paper.

\subsection{Necessary Condition for $k$-Vertex Global Rigidity}

In this subsection a necessary condition on $k$-vertex globally rigid graphs is provided which is a lower bound on the number of edges these graphs have. A prerequisite of the main theorem is to show that for fixed $k$, there always exists a $k$-vertex globally rigid graph of arbitrary size in which the number of edges depends linearly on the number of vertices.

Theorem 11. For ambient space dimension of $d=2$ and 3 , there exists a $k$-vertex globally rigid graph $G=(V, E)$ with $|V| \geq k+3$ and otherwise arbitrary, for which $|E|=a|V|+b$ holds, for some $a$ and $b$ dependent on $k$ and $d$ but independent of $|V|$.

Proof. The proof is by constructing a $k$-vertex globally rigid graph which satisfies the conditions of the theorem. First, observe that a complete graph $K_{k+d+1}$ is $k$-vertex globally rigid. The number of edges in $K_{k+d+1}$ is $m=$ $\left|V\left(K_{k+d+1}\right)\right|=\frac{(k+d)(k+d+1)}{2}$. Suppose $G_{1}=\left(V_{1}, E_{1}\right)$ and $G_{2}=\left(V_{2}, E_{2}\right)$ are two $K_{k+d+1}$ graphs. It is easy to show 
that by adding a set $L$ of $k+d+1$ edges between $G_{1}$ and $G_{2}$, such that every vertex in $G_{1}$ is connected to exactly 1 vertex in $G_{2}$ and conversely, $G=\left(V_{1} \cup V_{2}, E_{1} \cup E_{2} \cup L\right)$ is also $k$-vertex globally rigid.

More generally, to construct a $k$-vertex globally rigid graph $G=(V, E)$ on $n=|V|$ vertices imagine a series of $K_{k+d+1}$ graphs $\left\{G_{i}=\left(V_{i}, E_{i}\right)\right\}_{i=1 . .(p-1)}$ in which $G_{i}$ is connected to $G_{i+1}$ by $k+d+1$ edges, say $L_{i}$. Here, $p=\left\lfloor\frac{n}{m}\right\rfloor+1$ and $\left|V\left(G_{p}\right)\right|=n-p m \leq m$. For $G_{p}$, which may have fewer than $k+d+1$ vertices, connect every vertex to all vertices of $G_{p-1}$ (by $k+d+1$ edges), which constitute the set $L_{p-1}$ of edges between $G_{p-1}$ and $G_{p}$. It is easy to show that $G=(V, E)=\left(V_{1} \cup \ldots \cup V_{p}, E_{1} \cup \ldots \cup E_{p} \cup L_{1} \cup \ldots \cup L_{p-1}\right)$ is $k$-vertex globally rigid. Hence, the inequality $|E| \leq(p-$ $1) m+(p-1)(k+d+1)+(k+d+1) m$ holds. With $k$ fixed, $O(|E|)=O(p m)=O(n)$ holds, i.e. the number of edges is linear with respect to the number of vertices.

Theorem 12. In a strongly minimal $k$-vertex globally rigid graph the edge count is under-bounded by the formula $|E| \geq\left\lceil\frac{k+2}{2}|V|\right\rceil+c(k)$, where $c(k)$ is an integer $(c$ is independent of $|V|$ but depends on $k$ ) and if the equality holds (i.e. $|E|=\left\lceil\frac{k+2}{2}|V|\right\rceil+c(k)$ ), then $c(k) \geq 0$.

Proof. Assume that $G=(V, E)$ is a strongly minimal $k$ vertex globally rigid graph of $|V| \geq k+3$ vertices, whose number of vertices is $|E|=a|V|+c(k)$ (according to Theorem 11 such a graph exists), where $c(k)$ is independent of $|V|$. According to Lemma $2, \delta_{i} \geq k+2$ holds. Therefore, the average degree in $G$ is $\delta_{\text {avg }} \geq k+2$. On the other hand, $\delta_{\text {avg }}=\frac{2|E|}{|V|}=2 a+\frac{2 c(k)}{|V|}$. Hence, $2 a+\frac{2 c(k)}{|V|} \geq k+$ $2 \Rightarrow k \leq 2(a-1)+\frac{2 c(k)}{|V|}$.

Since the property must hold for graphs of arbitrary size and in particular arbitrarily large $|V|$, assuming $|V|>$ $2\left(c(k)\right.$, we will have $k \leq 2(a-1)$ or $a \geq \frac{k}{2}+1$. Therefore, $|E| \geq\left\lceil\left(\frac{k}{2}+1\right)|V|\right\rceil+c(k)$ holds for $|V|>2 c(k)$.

Now suppose that for some $|V|$ the equality holds (i.e. $\left.|E|=\left\lceil\frac{k+2}{2}|V|\right\rceil+c(k)\right)$. We prove that for such a strongly minimal $k$-vertex globally rigid graph $c(k) \geq 0$ always holds.

First suppose $k$ is even. Then, we will have $\delta_{\text {avg }}=k+2+$ $\frac{2 c(k)}{|V|} \geq k+2$, which implies $c(k) \geq 0$.

If $k$ is odd, then $|E|=\frac{k+1}{2}|V|+\left\lceil\frac{|V|}{2}\right\rceil+c(k)$ which gives $|E|<\frac{k+1}{2}|V|+c(k)+\frac{|V|}{2}+1$. Therefore, $\delta_{\text {avg }}<k+1+$ $\frac{2 c(k)}{|V|}+1+\frac{2}{|V|}$ holds. This implies $k+2<k+2+\frac{2 c(k)}{|V|}+\frac{2}{|V|}$ which gives $-1<c(k)$ and so $c(k) \geq 0$ holds.

Note that the underbound of Theorem 12 is achieved for $k=2$, see Theorem 7 . Below, we show it is also achievable for $k=3$.

\subsection{Strongly Minimal 3-vertex Global Rigidity in 2D}

In this section we provide a class of graphs that are strongly minimal 3-vertex globally rigid. According to the important result of Theorem 12, for the case of 3 vertex global rigidity, the number of edges must satisfy the inequality $|E| \geq\left\lceil\frac{5}{2}|V|\right\rceil+c$. In order to characterize a strongly minimal 3 -vertex globally rigid graphs, we

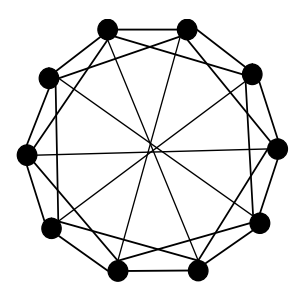

$\mathbf{a}$

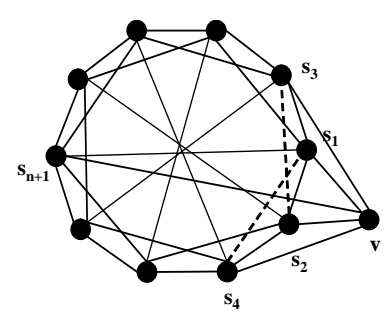

b
Figure 4. (a) A Strongly Minimal 3-Vertex Globally Rigid graph of size $10\left(R_{10}\right)$. (b) A Strongly Minimal 3vertex globally rigid graph of size $11\left(R_{11}\right)$.

conjecture that the number of edges has the form $|E|=$ $\left\lceil\frac{5}{2}|V|\right\rceil+c$ and then try to find a suitable value for $c$. We also assume that the number of vertices is at least 6 . Since we are seeking graphs with a minimum number of edges, it is reasonable to keep $c$ at its minimum (0) and try to find a graph which has the desired property.

If $|V|$ is even, i.e. $|V|=2 n$, the equation implies $|E|=$ $\frac{5}{2}|V|$ and $\delta_{\text {avg }}=5$. According to Lemma $2, \delta(v) \geq 5$. Therefore, the graph we are seeking, is 5-regular, i.e. every vertex has degree 5. Fortunately, there is a class of graphs with these conditions that are 3 -vertex globally rigid. An example of such a graph with $|V|=10$ is shown in Figure $4 \mathrm{a}$, and the structure carries over in an obvious way to graphs with $2 n$ vertices for $n \geq 3$. The structure of such a graph, call it $R_{2 n}$, is as follows:

The graph is formed from a $C_{2 n}$ cycle $\left[s_{1}, s_{2}, \ldots . s_{2 n}\right]$, to which are added the edges $s_{1} s_{3}, s_{2} s_{4}, s_{3} s_{5}, \ldots \ldots s_{2 n-1} s_{1}, s_{2 n} s_{2}$ (forming 2 cycles of size $n$ denoted by $C_{n}^{(1)}$ and $C_{n}^{(2)}$, respectively) and the edge set $D$ of edges $s_{i} s_{i+n}, i=1$..n (called diagonals). Note that the cycles $C_{n}^{(1)}$ and $C_{n}^{(2)}$ are disjoint.

It needs to be proved that the proposed graph is 3-vertex globally rigid. The proof has two steps: first, showing the graph is 5-connected, and second, after removal of any 2 vertices, showing the result is redundantly rigid. Proofs are omitted due to space limitation.

Theorem 13. The graph $R_{2 n}$ on 6 or more vertices, depicted in Figure 4a, is 5-connected.

Theorem 14. The graph $R_{2 n}$ on 6 or more vertices, is 3 vertex globally rigid.

To obtain a class of strongly minimal 3-vertex globally rigid with an odd number of vertices, i.e. $|V|=2 n+1$, we define an operation, termed 2-extension, over $R_{2 n}$ graphs to increase $|V|$ by 1 . We show that this operation preserves 3-vertex global rigidity. The sketch of 2-extension is as follows:

Assume vertices $s_{i}, i=1 . .4$ to be 4 consecutive vertices in the $C_{2 n}$ cycle as depicted in Figure $4 \mathrm{~b}$. The diagonal neighbor of $s_{1}$ is called $s_{n+1}$. The operation consists of adding a new vertex, say $v$, connecting it to $s_{i}, i=$ $1 . .4,(n+1)$ and removing $\left(s_{1}, s_{4}\right)$ and $\left(s_{2}, s_{3}\right)$.

Let $R_{2 n+1}$ be the graph obtained from $R_{2 n}$ by applying the 2-extension operation (Figure 4b). Evidently, 
Table 1. Comparison of the minimum number of required distance measurements for the suggested structures with $n$ vertices

\begin{tabular}{|c|c|}
\hline Structure & $\begin{array}{c}\text { Min \# of Dist. } \\
\text { Measurements }\end{array}$ \\
\hline 8-Connectivity & $8 n$ \\
\hline S.M. 3-V-G Rigid & $5 n$ \\
\hline
\end{tabular}

$\left|E\left(R_{2 n+1}\right)\right|=\left|E\left(R_{2 n}\right)\right|+3=\frac{5}{2}(2 n)+3=\frac{5}{2}(2 n+1)+\frac{1}{2}$ holds which is consistent with the edge count condition $\left(\left|E\left(R_{2 n+1}\right)\right|=\left\lceil\frac{5}{2}\left|V\left(R_{2 n+1}\right)\right|\right]=\frac{5}{2}\left(\left|V\left(R_{2 n+1}\right)\right|-1\right)+\frac{1}{2}\right)$. Hence, $R_{2 n+1}$ has the minimum number of edges and by proving that $R_{2 n+1}$ is 3 -vertex globally rigid, it follows that it is strongly minimal 3 -vertex globally rigid.

Theorem 15. The 2-extension operation applied to $R_{2 n}$ preserves 3 -vertex global rigidity.

Proof. The proof is similar to the proof of Theorem 14. The method is to consider all possible choices of $U$, $U \in V\left(R_{2 n+1}\right)$ and $|U|=2$, and show that $R_{2 n+1} \backslash U$ is globally rigid. We omit the proof due to the space limitation.

\subsection{Comparison of Results}

Table 1 shows the result of comparing the structures suggested for 3-vertex globally rigid graphs in Section 3.1 (8-connected) and Section 3.2 (strongly minimal). It is easy to see that the strongly minimal 3 -vertex globally rigid structure is at least $\frac{8 n-5 n}{8 n}=\frac{3}{8}=37.5 \%$ more efficient to localize the network (in terms of the minimum number of required distance measurements to achieve the specified tolerance to vertex loss), than the structure suggested based on connectivity. Of course, there will be other redundancies in the larger network which are not present in the strongly minimal network.

\section{CONCLUSION}

In this paper we studied the structure of localizable sensor networks which are tolerant against the loss of multiple nodes (the network remains localizable after the loss of multiple nodes). This is done by introducing the notion of $k$-vertex globally rigid graphs in which after removal of any set of up to $k-1$ vertices, the resulting network still remains globally rigid (localizable). For $2 D$ networks, we showed that a graph is $k$-vertex globally rigid if it is $(k+5)$-connected. For networks modeled by a random geometric graph, this reduction may enable us propose a critical transmission radius $r$ for the nodes, above which the network becomes $k$-vertex globally rigid. Furthermore, By providing a lower bound on the edge count of $k$-vertex globally rigid graph in terms of the vertex count, we also proposed a class of 3 -vertex globally rigid graphs with minimum edge count. Comparisons showed a considerable improvement achieved by this class over the 8-connectivity condition, in terms of the required number of distance measurements. This suggests that, there is certainly a benefit in studying the structure of strongly minimal networks for a given $k$ (and for $k>3$ this remains to be done). Indeed, the full characterization of (strongly minimal) $k$-vertex globally rigid graphs in addition to efficient algorithms to test this property on a given graph are still open problems that constitute our future work.

\section{REFERENCES}

Aspnes, J., Eren, T., Goldenberg, D., Morse, A., Whiteley, W., Yang, Y., Anderson, B., and Belhumeur, P. (2006). A theory of network localization. IEEE Transactions on Mobile Computing, 5(12), 1663-1678.

Connelly, R. (2005). Generic global rigidity. Discrete and Computational Geometry, 33(4), 549-563.

Graver, J., Servatius, B., and Servatius, H. (1993). Combinatorial rigidity. American Mathematical Society.

Jackson, B. and Jordan, T. (2005). Connected rigidity matroids and unique realizations of graphs. Journal of Combinatorial Theory, Series B, 94, 1-29.

Jackson, B. and Jordán, T. (2009). A sufficient connectivity condition for generic rigidity in the plane. Discrete Applied Mathematics, 157(8), 1965-1968.

Jordan, T. and Szabadka, Z. (2009). Operations preserving the global rigidity of graphs and frameworks in the plane. Computational Geometry, 42(6-7), 511-521.

Laman, G. (1970). On graphs and rigidity of plane skeletal structures. Journal of Engineering Mathematics, 4, 331340.

Lovasz, L. and Yemini, Y. (1982). On generic rigidity in the plane. SIAM Journal on Algebraic and Discrete Methods, 3, 91 .

Nagamochi, H. and Ibaraki, T. (2008). Algorithmic aspects of graph connectivity. Cambridge University Press New York, NY, USA.

Servatius, B. (1989). Birigidity in the plane. SIAM Journal on Discrete Mathematics, 2(4), 582-599.

Summers, T., Yu, C., and Anderson, B.D.O. (2008). Robustness to agent loss in vehicle formations and sensor networks. In Proceedings of the 47th IEEE Conference on Decision and Control, 1193-1199.

Wan, P. and Yi, C. (2004). Asymptotic critical transmission radius and critical neighbor number for $\mathrm{k}$ connectivity in wireless ad hoc networks. In Proceedings of the 5th ACM international symposium on Mobile ad hoc networking and computing, 1-8. ACM.

$\mathrm{Yu}$, C. and Anderson, B.D.O. (2009). Development of redundant rigidity theory for formation control. International Journal of Robust and Nonlinear Control, 19(13), 1427-1446.

Yu, C. and Anderson, B. (2008). Agent and Link Redundancy for Autonomous Formations. In Proceedings of the 17th IFAC World Congress, 6584-6589. IFAC.

Yu, C., Dusgupta, S., and Anderson, B.D.O. (2010). Robustness to agent loss in vehicle formations and sensor networks. Submitted for publication.

Yu, C., Fidan, B., and Anderson, B.D.O. (2006a). Principles to control autonomous formation merging. In Proceedings of the 2006 American Control Conference, 762-768. Minnesota USA.

Yu, C., Fidan, B., Anderson, B.D.O., and Hendrickx, J.M. (2006b). Merging multiple formations: A metaformation prospective. In In Proceedings of 45 th IEEE Conference on Decision and Control, 4657-4663. San Diego, CA. 